\title{
FAMILIAL HEMIPLEGIC MIGRAINE
}

\author{
BY \\ C. W. M. WHITTY \\ From the Department of Neurology, Radcliffe Infirmary, Oxford
}

The clinical phenomena of migraine are generally explained as due to vasoconstriction in some part of the internal carotid vascular system followed by vasodilatation in the territory of the external carotid vascular system and possibly of the internal also. On such a view the aura of migraine is supposed to be due to ischaemia and consequent dysfunction of part of the cerebral cortex, and the headache to tension in the walls of distended arteries. The evidence for vasodilatation as the cause of the headache is considerable, and ranges from such simple observations as its relief by pressure on the carotid artery and its branches to its reproduction by vasodilator drugs or artificial dilatation of vessel walls and its relief by vasoconstrictors (Wolff, 1948). The role of preceding vasoconstriction is not so well established, although the occasional direct observation of retinal artery spasm, the prolongation of the aura symptoms by vasoconstrictors, or the increase under their influence of electroencephalographic changes considered to represent cortical ischaemia, as well as the rarely observed relief of the aura by vasodilators, are arguments in favour. Such a theory does not attempt to explain the cause of migraine in the sense of what initiates the vascular changes themselves.

Some of the unusual forms of migraine are interesting both for the clinical picture they present and as a test of the validity of such a hypothesis as the above. Hemiplegic migraine is one such variety. Here the common visual aura may proceed to or be replaced by a hemiplegia (with aphasia if on the dominant side) and a partial hemi-anaesthesia, which is followed by a severe hemicranial headache. The occurrence " in some few cases ... of a transient hemiplegia "was mentioned by Liveing in 1873 in his extensive review of migraine, and since then a number of cases have been reported. The term has at times been rather loosely used to refer to any case in which the usual phenomena of migraine are associated with hemiplegia, even when not occurring as an aura to the attack. Some of these cannot be regarded as simple migraine, but appear to be examples of a migrainous syndrome symptomatic of an underlying structural pathology. Thus Ramsay Hunt (1915) discusses a patient who had had migrainous attacks since childhood. At the age of 32 she had one of her usual attacks with headache and vomiting, but this was accompanied by a brief period of loss of consciousness, and thereafter left hemiparesis with bilateral extensor plantar responses, severe neck stiffness, and papilloedema. At lumbar puncture the fluid contained fresh blood on repeated examinations. The pictureo suggested a leak from an angioma. The question of whether the preceding attacks of " normal , migraine were also linked with such a lesion mus $\frac{0}{3} \stackrel{9}{3}$ remain open in this case. However, the occurrence of recurrent focal neurological signs associated with severe lateralized headaches in known cases of cerebral angioma is recognized. Such cases mas at times cause diagnostic confusion, and it seems probable that they gave rise to the suggestion in the older literature that subarachnoid haemorrhage could occur as part of a simple migraine. The use of cerebral angiography has recently helped to clarify this point.

In other cases, though the initial signs and symptoms can be adequately explained by simple vasoconstriction, the subsequent course of the attack may suggest or even prove that a more permanent change such as thrombosis has occurred. In these cases the neurological signs may follow the headache or become aggravated rather than lessen as it develops. Oppenheim (1890) gives the case of a woman who had had migrainous headaches for many years. Four months after childbirth she had one of her usual attacks, which was accompanied by aphasia. Instead of clearing, a hemiplegia developed, and coma and death ensued. At necropsy there was extensive thrombosis of the left middle cerebral artery. Infeld (1901) describes a patient in whom an apparently typical attack was followed by hemiplegia and hemi-anaesthesia. These signs cleared partially but left a residue of paresthesiae, 
weakness, and athetotic movements in the affected hand. Von Schroeder (quoted by Hunt, 1915) mentions a patient who had severe hemicranial headaches for some years, and after a typical attack suddenly lost consciousness and on coming round had a hemiplegia and hemianopia. The paresis cleared but the hemianopia remained. Thomas (1907) also describes two cases of long-standing migraine with only visual aura, in each of which one attack was accompanied by hemiparesis, hemianaesthesia, and hemianopia. In each case all signs resolved except the hemianopia. Although some of these cases may represent the manifestations of a fixed lesion, such as an angioma, the occurrence of a permanent neurological deficit without such a structural vascular abnormality has been clearly demonstrated by Symonds (1952) in a case of migraine with visual aura. And the findings of Oppenheim leave no doubt that thrombosis in otherwise normal vessels can at times complicate an attack.

Apart from these cases, there are others in which a dense but completely reversible hemiplegia accompanies a migrainous attack. The neurological signs may be present for a few hours only or may persist for as long as a week, outlasting the headache, though they ultimately clear completely. These symptoms may alternate with attacks in which the migraine presents with some more usual and limited aura or simply as hemicrania and vomiting. There is also usually a family history of the complaint. Between attacks there are no neurological signs and health is normal. For all these reasons such. cases have justifiably been regarded as true hemiplegic migraine.

Jelliffe (1906) described two cases in some detail. In one, his second case, the possibility of a structural lesion arises, but his first case illustrates the syndrome. The patient had had attacks since childhood. The onset was with visual symptoms which proceeded to a right hemiparesis with sensory loss. Headache was severe, though described as mainly right-sided. All symptoms subsided completely in three to four days, and he was able to return to his normal routine. In 1910 Clarke published the history of a family of which three generations had suffered from the complaint. The attacks had a striking similarity from case to case. He gave details of six cases, though no less than 11 persons amongst 23 siblings were known to be affected. In all six the onset was with visual symptoms which proceeded to a hemiplegia with some degree of hemi-anaesthesia, and was followed in a few hours by severe hemicrania and vomiting when the neurological signs would begin to abate. In all the attacks detailed the signs were right-sided with aphasia, though in two cases attacks on the other side were mentioned. The attacks lasted two to three days and recovery was complete. They had occurred since childhood with a tendency to be less frequent as age advanced, his oldest patient being 54 years at his death from pneumonia. This description illustrates two points which are common in hemiplegic migraine; its strong familial tendency and the similarity in the pattern of attack within the family tree. Dynes (1939) also described the condition in a mother and daughter. Here the hemiparesis was either right- or left-sided, and again the course of the attack was the same in each case : signs would begin in the hand (and speech in right-sided attacks), proceed to a hemiparesis with some anaesthesia, but visual signs occurred relatively late. Symonds (1952) has added two further cases. In both there was a family history of similar attacks. In one, where the family history showed three generations affected, the patient had had a carotid angiogram done during the height of an attack. This had shown no abnormality. The electroencephalogram (E.E.G.) gave evidence of marked electrical abnormality throughout the right hemisphere. (The paresis was on the left.) The cerebrospinal fluid contained three polymorphs per c.mm. but was otherwise normal, though late in a previous attack there had been 185 polymorphs per c.mm. An air encephalogram done shortly after this had been normal. The case is of special interest as the first record of cerebral angiography done during an attack. As Symonds (1952) remarks, the evidence of its normality must exclude arterial spasm in any but the smallest arteries. Indeed, it is difficult to envisage a primary arterial spasm sufficient to cause an ischaemia which results in motor and sensory loss which may persist for days and yet recover completely. In this sense the "cause" of hemiplegic migraine remains a problem.

Four cases of the condition are briefly reported in this paper. Three illustrate the strongly familial tendency. In one, the opportunity arose to do both angiographic and E.E.G. studies, and the results are discussed. A fifth case, in which an isolated attack of hemiplegia followed by hemicrania occurred, is also described. Although it is doubtful whether the case could properly be considered one of hemiplegic migraine, it illustrates a possible mechanism whereby symptoms are produced, and seemed relevant and interesting.

Case 1 (R.I. 135977)._Familial hemiplegic migraine with prolonged confusion afterwards; pattern of attacks always the same with left-sided paresis.

A woman aged 52 had had " attacks" since the age of 
15, always of the same pattern. These were initiated by flashing lights in the left half-field, and normal vision in this field would be abolished. Soon afterwards numbness first of the left hand and then of the leg developed. She had difficulty with her speech; would use wrong words and " couldn't think of the words, though she knew what she wanted to say". In about two or three hours right-sided headache would develop and gradually increase in intensity; at this stage she occasionally vomited. The hemiplegia would clear slowly over a period of two or three days. As it was clearing she would often have visual hallucinations in the hemianopic field. She would see something moving and turn to find nothing there; occasionally she had formed hallucinations of a cat or someone's hand. She recognized them as hallucinatory but they worried her. She had never lost consciousness during an attack. Frequency of attack had varied, and she had been free for as long as five years. During the last nine years frequency had increased, and she would have them every two or three months. Two years before she was seen, on the assumption that the attacks were epileptic, she had been put on to a regime of phenobarbitone, "epanutin ", and "tridione". This had not reduced the attacks but had changed their character slightly. The initial visual phenomena persisted, but these were followed rapidly by severe headache, and the numbness and weakness of the limbs was much less prominent and of shortened duration. However, as this receded she would find that for as long as two weeks she was in a curious mental state in which she felt muddled and confused, could concentrate on nothing, had great difficulty in doing her housework, and was noticed by others to be irritable and argumentative. This would clear slowly, and she would feel, and seem to others, her normal self. She was admitted to hospital some five weeks after her last attack.

She was a cheerful, rather obese, woman of poor intelligence. She was right-handed for writing and sewing, though probably ambidextrous for cooking. One brother was considered to be left-handed. Her blood pressure was $200 / 110 \mathrm{~mm}$. $\mathrm{Hg}$, but fundal and peripheral vessels appeared healthy and urinary concentration was within normal limits. Radiographs of the skull were normal. An E.E.G. showed an alpha rhythm of 9 to 10 c.p.s. reduced on opening the eyes. There were paroxysms of high-voltage, 3 c.p.s. waves from both temporal areas, more marked on the right side and with some isolated spike discharges. Three days after admission a right carotid angiogram was done. This was normal. Some hours after this procedure she was noticed to be drowsy, irritable, and confused. In response to questions she appeared dysphasic. She had a left homonymous hemianopia, but denied any positive visual phenomena in the half field. There was a left hemiparesis, more marked in the arm, and anaesthesia to pin-prick and light touch. She complained of headache for the first time some 24 hours later, though she had probably had it for longer. During the next three days the signs slowly subsided, though some hemianopia persisted for five days. She remained somewhat confused for a week. The cerebrospinal fluid was examined on the second day of the attack. Pressure was $200 \mathrm{~mm}$., protein $50 \mathrm{mg}$. per $100 \mathrm{ml}$., and there were 10 polymorphs per c.mm. The Wassermann reaction was negative and the Lange curve 0000000000 . Electroencephalograms were done on the second, fourth, and eighth days after the onset of the attack. The first showed slow (less than 1 c.p.s.) medium voltage waves from the right hemisphere with no alpha rhythm; on the left side some 8 c.p.s. alpha waves were present, but this was interrupted by occasional high voltage 2 to 3 c.p.s. activity with some spikes. The further records showed a gradual return to the appearance in that made before the attack.

The family history was of interest. Her father had had similar attacks of left-sided paralysis with severe headaches throughout his life. In these he had occasionally lost consciousness and been incontinent. He was said to have died in an attack at the age of 61 . Her elder sister had also had frequent attacks of left hemiplegia with headaches but had never lost consciousness. A younger brother had not been affected at the time of his death in the 1914-18 war at the age of 25. An elder brother is Case 2 below. Of her four daughters aged $29,26,23$, and 11 , none have so far had attacks like hers. The second and youngest are entirely healthy, but the eldest and third have had " fainting fits " since childhood, sudden loss of consciousness with falling and marked pallor, but no involuntary movements or incontinence Electroencephalographic records were obtained from the second and third daughters. Both showed an unstable alpha rhythm with much 3 to 4 c.p.s. activity and some random spiking from the temporal areas. They resembled that of their mother between her attacks. This patien 8 showed a weak positive blood Wassermann reactions but the test in her brother and all the children wass negative.

Case 2 (R.I. 142521).-Familial hemiplegic migraine with occasional associated attacks of loss of consciousness; pattern of attacks always similar with left-sided paresis but occasional right-sided involvement also.

A man aged 56, the brother of Case 1, had suffered from severe right-sided headaches since the age of 17 . They occurred only about once a year, and were always associated with left-sided paresis. He would first notice some numbness and weakness of the left leg, this spread to involve the hand, and there was accompanying blurred vision, though he did not describe a clear-cut hemianopia. Headache then developed and built up gradually over some hours. During this time the weakness of the limbs increased, and very occasionally there was some weakness and clumsiness of the right hand also. He had noticed no speech difficulties, but felt drowsy and muddled and others had remarked on this. The headache usually cleared in 24 hours, but the weakness persisted for two or three days. On four occasions only he had had a sudden "blackout" in which he had lost consciousness, probably for a few minutes only, but he had never been incontinent. On coming round he had had the weakness noted above, and a headache soon developed. He regarded these as similar to his other 
attacks. On examination some months after his last attack there were no abnormal signs in the central nervous system. His blood pressure was $150 / 80 \mathrm{~mm}$. Hg. Radiographs of the skull were normal. The blood Wassermann reaction was negative. An electroencephalogram showed an unstable alpha rhythm with some bitemporal 6 c.p.s. activity and random spiking. Overbreathing caused the temporal waves to slow to 3 c.p.s., more markedly on the left side.

Case 3 (R.I. 165615).-Familial hemiplegic migraine with other forms of migraine also occurring in the patient and family.

A girl aged 16 had had episodic hemicranial headaches either right- or left-sided for three years. She had some difficulty in reading just before an attack, but the description did not suggest a localized field defect. For some hours before she would feel dull and lethargic, and would not go to work feeling that an attack was pending. These were her usual attacks, but on two occasions in the previous eight months, following the vague general symptoms, numbness and clumsiness of movement had developed in the left hand, spreading to the face and then to the leg. She could not walk properly or pick things up with that hand, and speech was slurred though not dysphasic. Right-sided headache developed in about half an hour, but the left-sided signs persisted for some hours. By the following morning she would be completely recovered. When examined some 10 days after her last attack there were no abnormal signs in the nervous system. A radiograph of the skull was normal. The E.E.G. record was abnormal. It showed diffuse 2,4 , and 6 c.p.s. waves in both hemispheres with occasional spike discharges. The abnormality was increased by overbreathing, but did not become paroxysmal. There were no localized abnormalities. Her mother, to whom she bore a striking physical resemblance, had had similar attacks for many years.

Case 4 (R.I. 133116).-Hemiplegic migraine with other forms of migraine also occurring in the patient, and a family history of the latter.

A woman university student aged 19 had suffered from migraine since the age of 12 . Her usual attacks were initiated by a general blurring of vision. Things appeared to be rippling as if seen through water. Headache, usually left- but sometimes right-sided, would follow in about half an hour and would increase in severity and lead to vomiting. Sometimes, though rarely, after the visual symptoms, paresthesiae in the right hand and aphasia would develop; weakness of the arm and leg would follow. These symptoms would persist after the onset of headache, but gradually decreased during the next few hours. On one occasion some weakness and numbness of the hand had persisted for six days. When seen at this time there was a significant reduction in tendon jerks in the right arm, some change in the appreciation of light touch and pin-prick in the hand, but no actual sensory loss. The condition cleared completely in the next few days. Radiographs of the skull were normal. The E.E.G. record showed some 4 and 5 c.p.s. activity in both temporal regions.
This was increased by overbreathing, but did not become paroxysmal. Her father had migraine, but with a visual aura only.

This patient, an intelligent and reliable observer, had taken ergotamine tartrate for a time with considerable relief of the headaches, but had abandoned it in favour of aspirin and codeine because she considered that ergotamine prolonged the aura.

Case 5 (S. M. S/1532). - A clincal syndrome of hemiplegic migraine associated with an allergic reaction to fish : no family history.

A man aged 34 eight days before he was seen had eaten some fish which disagreed with him. During the following day he had nausea and diarrhoea. The following morning, 36 hours after, he developed a very irritating urticaria over the chest and arms, but he proceeded to work as usual. Shortly after starting out he felt sick and vomited once or twice. He had a slight generalized headache, and then noticed that he could not see clearly to the right. The right arm felt numb and tingling, the leg was also affected, and on attempting to walk he fell down. His speech became thick and he could not say what he wanted to. There was no loss or clouding of consciousness, and he was fully aware that his mates helped him to an ambulance and that he was taken to hospital. He noticed at this time a gradually increasing, throbbing, left-sided headache. This largely cleared in the next two days but the hemiparesis persisted for four to five days, clearing slowly. When first examined in hospital a right hemiparesis including the face, with increased reflexes and extensor plantar response, was noted. There was diminished sensation to pin-prick and light touch, and a slight dysphasia. The cerebrospinal fluid was of normal pressure with $30 \mathrm{mg}$. protein per $100 \mathrm{ml}$. and 1 lymphocyte per c.mm. Seven days later there was some increase of reflexes on the right side but no other abnormality. When examined three weeks after the attack the nervous system was entirely normal. At this time radiographs of the skull were normal. The E.E.G. record showed a stable 8 c.p.s. alpha rhythm. There were a few sharp waves from both occipital regions which were increased but did not become paroxysmal on overbreathing. The record was almost within normal limits.

Previous history revealed a severe urticarial reaction following an A.T.S. inoculation eight months earlier, and frequent attacks of asthma and bronchitis as a child. He had occasional retro-orbital throbbing headaches, sometimes severe, but never sufficient to keep him from work. There was no family history of migraine or unusual allergic manifestations

\section{Discussion}

A survey of the cases reported here and those in the literature suggests that they fall into two rather dissimilar groups. In one the course of events is more like that of the more usual forms of migraine, an aura phase in which the hemiplegia appears and is followed shortly by headache, when the paresis slowly subsides. Attacks may be either 
right- or left-sided, and migraine with a more limited aura may also occur in the same patient. The family history usually reveals other cases of migraine, but they may not be always of the hemiplegic variety. In the other group the attacks are more stereotyped. Often they occur constantly on one side, and other varieties of attack in a given case are unusual. The signs of the aura are prolonged, sometimes for days, and may persist or increase after the onset of headache. The cerebral disturbance is more widespread ; confusion, drowsiness, and even coma may be noted; sometimes motor signs may for a while be bilateral. The family history is very striking. Not only are many individuals in several generations affected, but the detailed pattern of attack is unusually constant from case to case. It is for this group particularly that the term familial hemiplegic migraine seems appropriate. The syndrome is well illustrated in Clarke's patients, and in the first, second, and possibly third patients of this series.

The first two cases and their families raise points of special interest. They provide evidence both clinical and electroencephalographic of a hereditary " cerebral dysrhythmia" which may show itself as attacks of loss of consciousness. There is also evidence of a hereditary factor responsible for a remarkably stereotyped pattern of migraine. It would be tempting to associate the two and suggest that the vascular spasm element of the migraine also precipitates an attack of loss of consciousness. This, however, is clearly not so. For in Case 2 and his father the two types of attack are associated in the same individual, while in Case 1 and her sister only migrainous attacks occurred, and in two of her children only loss of consciousness. Further, in one child a "familial " electroencephalographic abnormality was shown, though neither type of attack had as yet occurred. The two tendencies seem to be quite separate here, and capable of independent inheritance, even if some more intimate link between epilepsy and migraine in general is indicated by other cases.

Case 1 also provides some suggestive evidence in favour of arterial spasm as responsible for the onset of her attacks. Angiography is known at times to cause arterial spasm in the territory of the injected vessel, especially if hypertension be present, and angiography in this case clearly precipitated an attack. Why the spasm should start, as the clinical onset of visual symptoms suggests it so frequently does, in the posterior cerebral circulation, remains a problem. It has been shown by Moore and Stern (1938) that the anatomical course of the posterior cerebral artery allows it to be readily compressed against the edge of the tentorium. But this requires some rise in supratentorial pressure to bring it about, of which there is no clear evidence in the cases under discussion. Such an anatomical factor, though possible, must therefore remain hypothetical. Indeed, it is difficult to see how simple spasm could be responsible for signs which persisted for five days and then disappeared. That relative ischaemia can cause loss of neuronal function short of cell necrosis is accepted, but if the vascular spasm responsible for this is brief, then recovery should be more rapid. If it lasts for days, then permanent damage should result. The explanation put forward by Symonds (1952) for such a prolongation of signs is that small thromboses may occur in the terminal arterioles during the spastic phase, and in support of this he cites the polymorph pleocytosis in the cerebrospinal fluid of one of his cases. But such a hypothesis also requires that some permanent damage should occur. Some slight reduction in the cerebral cell population must result on each occasion. He reçognizes this difficulty, and suggests that the gradual mental deterioration recorded in one such case does, in fact, represent this permanent 5 deficit. However, if the recurrent signs are always hemiplegic and on the same side, it seems surळ $N$ prising that some slight permanent lateralize disability does not result. In one of Clarke's cases and in two of those described here attacks had been occurring mainly on one side for decades, bug no neurological sequelae could be found. It possible that after arterial spasm there is som $\overrightarrow{0}$ consequent oedema in the area supplied when bloot $\mathrm{C}$ flow is restored just as in a limb temporarily deprived of its arterial blood. This could account for a prolongation of signs, and would suggest a mechanism similar to that envisaged in " angioneurotic oedema" of the brain. Such a process would explain the more widespread disturbances sometimes noted, and would lead to less permanent damage than recurrent small thromboses.

Some such mechanism is probably illustrated in Case 5. He would hardly qualify as one of hemiplegic migraine, though the symptoms are strikingly similar. In him, their mechanism of production was presumably " allergic". A condition of oedema similar to the urticaria of the skin may be supposed to have occurred also in parts of the left cerebral hemisphere. Such a "cause " for migraine has frequently been postulated. Osler (quoted by Russell, 1909) cites the case of a boy aged 12 who had occasional attacks of right hemiplegia with aphasia lasting for some days but showing complete recovery. The attacks diminished in severity and at the age of 26 he developed severe migraine, and 
at 28 started to have attacks of urticaria and angioneurotic oedema. Osler suggested that the earlier hemiplegic attacks and the migraine were caused by "angioneurotic oedema of the brain ". Foster Kennedy $(1926,1936)$ assembled a number of cases of episodic focal neurological abnormalities in which the evidence for an allergic precipitation seemed convincing. In some, for instance, eliminating certain articles of diet, for which positive skin sensitivity tests had been obtained, completely abolished the attacks. Amongst them were cases which presented as migraine with a focal aura, and at least one which could be regarded as a typical hemiplegic migraine. That increased intracranial tension can accompany such supposedly allergic and reversible neurological lesions seems clear from the observation of a case where an urticarial reaction to injected serum was accompanied by headaches, neck stiffness, papilloedema, hemiparesis, and increased C.S.F. pressure. As the urticaria subsided, so the neurological picture cleared (Foster Kennedy, 1929). However, that such increase is due to local or general brain swelling, rather than to extracerebral vascular factors, remains an assumption. It seems unlikely that all cases are of this type. Nevertheless it may be the explanation in some. If so, then evidence of raised intracranial pressure might be expected. This was apparently not so in cases 1 and 5 of this series, where cerebrospinal fluid pressure was measured during an attack, nor in Symonds' second case. It is possible that brain swelling may occur at the expense of the volume of the cerebrospinal fluid so that the general rise in intracranial pressure may be slight. However, a more likely explanation is that oedema, if present, is localized. In that case it is the strategic siting of the oedema which produces the signs of cerebral dysfunction, and total intracranial volume may be little if at all increased. The phrase " hives of the brain ", attributed to Osler, may be an apt description of the intracranial state. In many ways the conception of a reversible focal oedema of the brain seems to fit better than simple ischaemia from vascular spasm, with a clinical picture in which neurological dysfunction of fairly rapid onset may persist for days, and yet finally resolve completely.

I am grateful to Sir Charles Symonds for some helpful comments on this paper. My thanks are also due to Dr. J. D. Kidd and Dr. M. S. Good for the opportunity of seeing two of these cases.

\section{REFERENCES}

Clarke, J. M. (1910). Brit. med. J., 1, 1534.

Dynes, J. B. (1939). Ibid., 2, 446.

Hunt, J. R. (1915). Amer. J. med. Sci., 150, 313.

Kennedy, F. (1926). Arch. Neurol. Psychiat., Chicago, 15, 28.

- (1929). Amer. J. med. Sci., 177, 555. (1929). Amer. J. med. Sci., 177, 555.

Infeld, M. (1901). Wien. klin. Wschr., 14, 673

Jelliffe, S. E. (1906). N. Y.Med. J., 83, 33.

Liveing, E. (1873). On Megrim, Sick-Headache, and Some Allied Disorders. Churchill, London.

Moore, M. T., and Stern, K. (1938). Brain, 61, 70.

Oppenheim, H. (1890). Charité-Ann., 15, 298.

Osler, W. (1909). Quoted by Russell, A. E. Clin. J., 34, 414

Schroeder, J. von (1884). Klin. Mbl. Augenheilk., 22, 351 .

Symonds, C. (1952). Trans. med. Soc. Lond., (1950-51), 67, 237.

Thomas, J. J. (1907). J. nerv. ment. Dis., 34, 153.

Wolf, H. G. (1948). Headache and Other Head Pain. Oxford University Press, New York.

\section{A P P E N D I X}

Since this paper was written, a further case (R. I. 167618) of the familial type has been seen in a man of 39. The hemiplegia was always right-sided. A left carotid angiogram, which was normal, again appeared to precipitate a typical attack which followed about one hour after the procedure. This hemiparesis was marked for 48 hours and cleared gradually in the next 24 hours. About 20 hours after its onset electroencephalograms, lumbar puncture, and air-encephalograms were done. The E.E.G. record, which had previously shown a stable alpha rhythm, blocked on opening the eyes, now showed almost complete disappearance of alpha. There were also short runs of 5 to 6 c.p.s. waves in the temporal and frontal leads, more on the left than on the right, increased on overbreathing. The cerebrospinal fluid, previously showing 4 lymphocytes per c.mm. and a protein of $45 \mathrm{mg}$. per $100 \mathrm{ml}$. with a pressure of 140 , now had 84 polymorphs and 40 lymphocytes per c.mm. with a protein of $35 \mathrm{mg}$. per $100 \mathrm{ml}$. Pressure was not measured as the patient was in the sitting position, but rate of flow did not suggest any marked rise. Air pictures showed no localized distortion or displacement of the ventricular system. The left lateral ventricle was slightly larger than the right, but this was within the range of physiological asymmetry occasionally seen. This finding seems to exclude any degree of generalized left hemisphere oedema, though it is not incompatible with focal. Indeed, the cerebrospinal fluid changes, similar to those observed by Symonds (1952), must reflect some change of an exudative nature in the cortex. 